PROCEEDINGS OF THE

AMERICAN MATHEMATICAL SOCIETY

Volume 130, Number 2, Pages 585-590

S 0002-9939(01)06106-8

Article electronically published on June 8, 2001

\title{
GROWTH OF FUNDAMENTAL GROUPS AND ISOEMBOLIC VOLUME AND DIAMETER
}

\author{
OGUZ C. DURUMERIC
}

(Communicated by Wolfgang Ziller)

\begin{abstract}
Some properties of fundamental groups of Riemannian manifolds $M$ will be studied without a lower bound assumption on Ricci curvature. The main method is to relate the local packing to global packing instead of using the Bishop-Gromov relative volume comparison. This method allows us to control the volume growth of the universal cover $\tilde{M}$ and yields bounds on the number of generators of $\pi_{1}(M)$ in terms of some isoembolic geometric invariants of $M$.
\end{abstract}

\section{INTRODUCTION}

In this article, we discuss the number of generators and the growth of the fundamental groups of Riemannian manifolds without curvature restrictions.

Let $M^{n}$ denote a compact $n$-dimensional Riemannian manifold without boundary, and let $\pi_{1}(M, p), d(M), v(M)$, and $i(M)$ denote its fundamental group, diameter, volume, and injectivity radius, respectively. For a finitely generated group $G$, $m g(G)$ denotes the minimal number of elements needed to generate the group. The entropy $h(G)$ of a group is a measurement of the number of distinct words (of a given length or less) in terms of generators and their inverses, and the entropy of a manifold $h(M)$ is a measurement of the volume growth of its universal cover; see Section 2 or $[\mathrm{Gr}$ ] for their formal definitions.

There are several studies of the fundamental group with lower bounds on Ricci curvature. Bishop(-Gromov) volume comparison is a basic tool for comparing the volumes of metric balls used in these results. In Milnor [Mi], the fundamental groups of manifolds with nonnegative Ricci curvature are shown to have polynomial growth, in contrast to negative sectional curvature cases in which they have exponential growth. The positive and nonnegative Ricci curvature cases are studied further by Anderson $[\mathrm{A}$, and the general case with an arbitrary lower bound on Ricci curvature is studied qualitatively by Gromov [Gr, chap. 6]. The case of negative or nonpositive sectional curvature has been studied extensively; see Preismann $[\mathrm{P}$, Gromoll-Wolf [GW], and Lawson-Yau [LY].

Definition 1.1. The isoembolic volume is defined to be $V_{e}\left(M^{n}\right)=v(M) / i(M)^{n}$ and isoembolic diameter to be $d_{e}\left(M^{n}\right)=d(M) / i(M)$ for an $n$-dimensional Riemannian manifold $M$.

Received by the editors July 31, 2000.

2000 Mathematics Subject Classification. Primary 53C20, 53C23.

Key words and phrases. Isoembolic, fundamental group. 
Let $E_{N}^{n}=\left\{M^{n}: V_{e}(M) \leq N\right\}$. The following results show that a bound on the isoembolic volume puts a priori topological and geometric restrictions on the manifolds. In [Be], Berger proved that $V_{e}\left(M^{n}\right) \geq V_{e}\left(S^{n}(1)\right)$ and equality holds if and only if $M^{n}$ is isometric to a standard sphere $S^{n}(r)$ of dimension $n$ with radius $r$. By the work of Croke [C2, $M^{n}$ is homeomorphic to a sphere $S^{n}$, if $V_{e}\left(M^{n}\right) \leq V_{e}\left(S^{n}(1)\right)+c(n)$ for a universal constant $c(n)$. In 1988, Yamaguchi proved that $E_{N}^{n}$ has finitely many homotopy equivalence types, $[\mathrm{Y}$. By the author's work [D2, one can estimate a priori upper bound for the number of homotopy types and the Betti numbers $b_{k}(M, \mathbf{F})$ in terms of $V_{e}\left(M^{n}\right)$ for any field $\mathbf{F}$. In particular, $b_{1}(M, \mathbf{F}) \leq C(n) V_{e}\left(M^{n}\right)^{2}$ for a universal constant $C(n)$, and a linear inequality is not possible, D2.

Even though $m g\left(\pi_{1}(M, p)\right)$ are uniformly bounded on $E_{N}^{n}$, one cannot deduce a priori upper bounds on $m g\left(\pi_{1}(M, p)\right)$ from the results above. In particular, the inequality $m g\left(\pi_{1}(M, p)\right) \geq b_{1}(M, \mathbf{F})$ by the Hurewicz Isomorphism Theorem is in the opposite direction.

Theorem 2. For any compact Riemannian manifold $M^{n}$,

$$
m g\left(\pi_{1}(M, p)\right) \leq\left(c V_{e}(M)\right)^{14 d_{e}(M)} \leq\left(c V_{e}(M)\right)^{4 c V_{e}(M)}
$$

and

$$
h\left(\pi_{1}(M, p)\right) \leq 14 d_{e}(M) \log c V_{e}(M)
$$

where $c$ is a constant depending only on the dimension $n$.

Theorem 2 is a consequence of Theorem 1 which involves controlling the volume growth of the universal cover by using packing arguments without a lower bound on the Ricci curvature. Here we briefly introduce the notation and definitions needed to state Theorem 1. The formal definitions will be given in Section 2. $B(p, r)$ denotes the metric ball of radius $r$ at $p$. The packing number $N(a, c, M)$ is the largest number of disjoint open metric balls of radius $c$ that can be fitted into any metric ball of radius $a$ in $M$. N $(a, c, M)$ obviously bounds $N(b, c, M)$, if $b \leq a$. The crucial Proposition 3.1 is about controlling $N(a, c, M)$ in terms of $N(b, c, M)$ for $6 c<b \leq a$.

Theorem 1. Let $M^{n}$ be a complete Riemannian manifold of dimension $n$ with the Riemannian universal covering map $\Psi: \tilde{M} \rightarrow M$. Let $R$ be such that $\forall p \in M$, $B(p, R, M)$ is simply connected in $M$; that is, every closed curve in $B(p, R, M)$ is contractible in $M$. Let $N(R, R / 7 ; M)=N_{0}$. Then, for $\Psi(\tilde{p})=p$,

$$
\operatorname{card}\left(\Psi^{-1}(p) \cap \bar{B}(\tilde{p}, a, \tilde{M})\right) \leq N_{0}^{(7 a / R)-5}, \quad \forall a>R .
$$

Consequently, if $M^{n}$ is compact with diameter $d(M)$, then

$$
m g\left(\pi_{1}(M, p)\right) \leq N_{0}^{14 d(M) / R}
$$

and the entropies satisfy

$$
h(M) \leq 7\left(\log N_{0}\right) / R
$$

and

$$
h\left(\pi_{1}(M, p)\right) \leq 14\left(\log N_{0}\right) d(M) / R .
$$




\section{BASIC NOTATION AND DEFINITIONS}

We refer to $\mathrm{BC}$, $\mathrm{CE}$ and $\mathrm{GKM}$ for basic Riemannian geometry. Let $\left(M^{n}, g\right)$ denote an $n$-dimensional compact Riemannian manifold unless otherwise specified. $d_{M}(.,$.$) is the Riemannian distance function. For X \subset M$, let $\operatorname{card}(X), X^{\circ}, \bar{X}$ and $v(X)$ denote the cardinality, interior, closure and Riemannian volume of $X$.

Definition 2.1. (i) $B(p, r)=\left\{x \in M: d_{M}(x, p)<r\right\}$ and $\bar{B}(p, r)=\{x \in M$ : $\left.d_{M}(x, p) \leq r\right\}$.

(ii) The packing number $N(a, c, M)$ is defined to be the supremum of the number of disjoint open metric balls of radius $c$ inside each open metric ball of radius $a$ in $M$.

Definition $2.2(\underline{\mathrm{Gr}}, 5 \mathrm{~B}])$. Let $G$ be a finitely generated group.

(i) The minimal number of generators is defined to be $m g(G)=\inf \{\operatorname{card}(H)$ : $H \subset G$ and $H$ generates $G$.

(ii) The entropy of a finite generating set $H$ of $G$ is $h(H)=\liminf _{t \rightarrow \infty} \frac{\log N(t, H)}{t}$, where $N(t, H)$ is the number of distinct words of length at most $t$ by using generators from $H$ and their inverses. The entropy of $G$ is $h(G)=\inf _{H} h(H)$, where $H$ runs through the generating subsets of $G$.

(iii) Let $M^{n}$ be a complete manifold of dimension $n$, with the Riemannian universal covering map $\Psi: \tilde{M} \rightarrow M$. The entropy of $M$ is defined to be $h(M)=$ $\liminf \frac{\log (v(B(\tilde{p}, t, \tilde{M})))}{t}$; that is, a measurement of the volume growth of the universal cover.

Definition 2.3. Let $A \subset B$ be both connected and let $i$ be the inclusion map. $A$ is said to be simply connected in $B$, if every closed curve in $A$ is contractible in $B$; that is, $i_{*}\left(\pi_{1}(A, a)\right)=0$.

\section{Proofs of the theorems}

Proposition 3.1. For any complete Riemannian manifold $M$ and $\forall a \geq b>6 c>$ 0 , one has $N(a, c, M) \leq N(b, c, M)^{k}$, where $k$ is the smallest integer $\geq \frac{\bar{a}-6 c}{b-6 c}$.

Proof. Let a metric ball $B(p, a)$ and a maximal family $\mathcal{F}=\{B(q, c): q \in Q\}$ of disjoint open balls inside $B(p, a)$ be given. Define

$$
Q(R)=\{q \in Q: B(q, c) \subset B(p, R)\}
$$

for $R>0$. Fix any $R$ with $b \leq R \leq a$, and choose any $q \in Q(R+b-6 c)-Q(R)$. Let $p^{\prime}$ be a point on any minimal geodesic from $p$ to $q$ such that $d\left(q, p^{\prime}\right)=b-3 c$, so that $d\left(p, p^{\prime}\right)<R-3 c$ and $B\left(p^{\prime}, 2 c\right) \subset B(p, R-c)$. There exists a $q^{\prime}$ in $Q \cap B\left(p^{\prime}, 2 c\right)$, for otherwise, the addition of $B\left(p^{\prime}, c\right)$ to $\mathcal{F}$ would contradict the maximality of $\mathcal{F}$. Furthermore,

$$
\begin{gathered}
d\left(p, q^{\prime}\right) \leq d\left(p, p^{\prime}\right)+d\left(p^{\prime}, q^{\prime}\right) \leq R-c, \text { hence } B\left(q^{\prime}, c\right) \subset B(p, R) \text { and } q^{\prime} \in Q(R), \\
d\left(q, q^{\prime}\right) \leq d\left(q, p^{\prime}\right)+d\left(p^{\prime}, q^{\prime}\right) \leq b-c, \text { hence } B(q, c) \subset B\left(q^{\prime}, b\right), \\
\forall q \in Q(R+b-6 c)-Q(R) \exists q^{\prime} \in Q(R) \text { such that } B(q, c) \subset B\left(q^{\prime}, b\right), \\
\operatorname{card}(Q(R+b-6 c)-Q(R)) \leq \operatorname{card}(Q(R))(N(b, c)-1), \\
\operatorname{card}(Q(R+b-6 c)) \leq \operatorname{card}(Q(R)) N(b, c) .
\end{gathered}
$$


Since $\operatorname{card}(Q(b)) \leq N(b, c)$, it follows that $\operatorname{card}(\mathcal{F})=\operatorname{card}(Q(a)) \leq N(b, c)^{k}$, where $k$ is the smallest integer $\geq \frac{a-b}{b-6 c}+1=\frac{a-6 c}{b-6 c}$.

$p$ is arbitrary and $\mathcal{F}$ is maximal, hence $N(a, c, M) \leq N(b, c, M)^{k}$.

Proof of Theorem 1. Let $\Psi: \tilde{M} \rightarrow M$ be the Riemannian universal covering map and $G=\pi_{1}(M, p)$. Choose any $\tilde{p} \in \Psi^{-1}(p)$ and let $G=\left\{g_{i}: i \in \Lambda\right\}$ act on $\Psi^{-1}(p)=\left\{g_{i} \tilde{p}: i \in \Lambda\right\}$ by deck transformations.

The following basic facts which were used or proved in [Mi] and [Gr, chap. 5] can also be found in [D1]. $W=M-\operatorname{cutlocus}(p)$ is an open, dense and contractible subset of $M$, [E]. $\forall g_{i} \in G, \exists$ an open contractible subset $W_{i}$ of $\tilde{M}$ such that $g_{i} \tilde{p} \in W_{i}$, $\Psi \mid W_{i}$ is an isometry of $W_{i}$ onto $W, W_{i} \cap W_{j}=\emptyset$ if $g_{i} \neq g_{j}, v\left(W_{i}\right)=v(M)$, and $\bigcup_{i \in \Lambda} \bar{W}_{i}=\tilde{M}$. If $M$ is compact, then $H=\left\{g_{i} \in G: \bar{W}_{i} \cap \bar{W}_{0} \neq \emptyset\right\}$ is a generating set for $G$, where $\tilde{p} \in W_{0}$. For compact $M, \forall g_{i} \in H, g_{i} \tilde{p} \in \bar{B}(\tilde{p}, 2 d(M) ; \tilde{M})$.

If $A$ is a path-connected subset of $\tilde{M}$ and $\Psi \mid A$ is not one-to-one, then $\Psi(A)$ contains a closed curve $\gamma$ which has a non-closed lift to $\tilde{M}$ and $0 \neq[\gamma] \in \pi_{1}(M, q)$. By the hypothesis, $\forall r \leq R$ and $\tilde{q} \in \tilde{M}, \Psi \mid B(\tilde{q}, r ; \tilde{M})$ is one-to-one and an isometry, and $v(B(\tilde{q}, r ; \tilde{M}))=v(B(\Psi(\tilde{q}), r ; M)$.

$\operatorname{card}\left(\Psi^{-1}(p) \cap \bar{B}(\tilde{p}, a ; \tilde{M})\right)=1$, if $0<a<R$.

Let $a \geq R$ be fixed, set $c=\frac{R}{7}$ and choose a maximal family $\mathcal{F}=\{B(q, c): q \in Q\}$ of disjoint open balls inside $B(\tilde{p}, a+c ; \tilde{M})$. From the maximality of $\mathcal{F}$, it follows that $\bar{B}(\tilde{p}, a ; \tilde{M}) \subset \bigcup_{q \in Q} B(q, 2 c, \tilde{M})$. Since the restrictions of $\Psi$ to metric balls of radius $R$ are isometries, $N(R, c ; \tilde{M})=N(R, c ; M)=N_{0}$. Given two distinct elements $g_{1} \tilde{p}$ and $g_{2} \tilde{p}$ of $\Psi^{-1}(p) \cap \bar{B}(\tilde{p}, a ; \tilde{M})$, one has $g_{1} \tilde{p} \in B\left(q_{i_{1}}, 2 c ; \tilde{M}\right)$ and $g_{2} \tilde{p} \in B\left(q_{i_{2}}, 2 c ; \tilde{M}\right)$ for $q_{i_{1}} \neq q_{i_{2}}$ of $Q$, otherwise, $\Psi \mid B\left(q_{i_{1}}, 2 c ; \tilde{M}\right)$ will not be one-to-one. Hence, $\forall q \in Q, B(q, 2 c, \tilde{M})$ contains at most one element of $\Psi^{-1}(p) \cap \bar{B}(\tilde{p}, a ; \tilde{M})$. By Proposition 3.1,

$$
\begin{aligned}
\operatorname{card}\left(\Psi^{-1}(p) \cap \bar{B}(\tilde{p}, a ; \tilde{M})\right) & \leq \operatorname{card}(Q) \leq N(a+c, c, \tilde{M}) \\
& \leq N(R, c, \tilde{M})^{\frac{a+c-6 c}{R-6 c}} \leq N_{0}^{\frac{7 a}{R}-5} .
\end{aligned}
$$

If $M$ is compact, then $\pi_{1}(M, p)$ can be generated by loops of length at most $2 d(M)$ by above. By taking $a=2 d(M)$ in the above inequality yields

$$
m g\left(\pi_{1}(M, p)\right) \leq N_{0}^{14 d(M) / R} .
$$

Any word of length at most $k$ constructed by using loops of length at most $2 d(M)$ has a lift to the universal cover starting at $\tilde{p}$ and with length at most $2 k d(M)$. So, the end point is in $B(\tilde{p}, 2 k d(M) ; \tilde{M})$. This proves that

$$
h\left(\pi_{1}(M, p)\right) \leq 14 d(M)\left(\log N_{0}\right) / R .
$$

For the entropy of $M, h(M) \leq 7\left(\log N_{0}\right) / R$ follows

$$
\begin{aligned}
v(B(\tilde{p}, a ; \tilde{M})) & \leq \operatorname{card}\left(\Psi^{-1}(p) \cap \bar{B}(\tilde{p}, a+d(M) ; \tilde{M})\right) v(M) \\
& \leq N_{0}^{7(a+d(M)) / R} v(M) .
\end{aligned}
$$

Proposition 3.2. Let $R, V, v>0$ be given. For any compact Riemannian manifold $M$, if $v(M) \leq V, \forall p \in M, B(p, R)$ is contractible in $M$, and $v(B(p, R / 7)) \geq v$, then $m g\left(\pi_{1}(M, p)\right) \leq L^{14 d(M) / R} \leq L^{4 L}$, where $L=V / v$. 
Proof. Clearly, $N_{0}=N(R, R / 7 ; M) \leq \frac{V}{v}=L$. Let $p$ and $q$ be a pair of furthest points of $M$, that is, $d(p, q)=d(M)$. Choose any normal geodesic $\gamma$ from $p$ to $q$, with $\gamma(0)=p$. Let $p_{i}=\gamma((2 i-1) R / 7)$ for $1 \leq i \leq s$ so that $d\left(p_{s}, q\right)<R / 7$ and hence $\frac{2}{7} s R \geq d(M)$. The balls $B\left(p_{i}, R / 7\right)$ are disjoint, since a nonempty intersection of $B\left(p_{i}, R / 7\right)$ and $B\left(p_{j}, R / 7\right)$ contradicts the minimality of $\gamma$ between $p_{i}$ and $p_{j}$. This implies $s v \leq V$. By Theorem 1 ,

$$
m g\left(\pi_{1}(M, p)\right) \leq\left(\frac{V}{v}\right)^{14 d(M) / R} \leq\left(\frac{V}{v}\right)^{4 s} \leq\left(\frac{V}{v}\right)^{4 \frac{V}{v}}=L^{4 L} .
$$

Proof of Theorem 2. If $r \leq i(M) / 2$, then $v\left(B\left(p, r ; M^{n}\right)\right) \geq r^{n} 2^{n-1} \alpha_{n-1}^{n} n^{-n} \alpha_{n}^{1-n}$ by Croke [Cr], where $\alpha_{n}=v\left(S^{n}(1)\right)$. Hence, by taking $v(M)=V$ and $R=i(M)$, one uses $\frac{d(M)}{R}=d_{e}(M)$ and $N_{0} \leq \frac{V}{v}=L \leq V_{e}(M) c$ in Proposition 3.2 to conclude the proof, where $c=2(7 / 2)^{n} \alpha_{n-1}^{-n} n^{n} \alpha_{n}^{n-1}$.

The method we developed above yields the following for noncompact manifolds.

Theorem 3. Let $M$ be a noncompact Riemannian manifold and $G_{A}$ be any subgroup of $\pi_{1}(M, p)$ generated by loops of length at most $A$. Let $R$ be such that $\forall p \in$ $B(p, A, M), B(p, R, M)$ is simply connected in $M$. Let $N_{0}=N(R, R / 7 ; B(p, A, M))$. Then

$$
m g\left(G_{A}\right) \leq N_{0}^{7 A / R} \text { and } h\left(G_{A}\right) \leq 7 A\left(\log N_{0}\right) / R .
$$

The proof of Theorem 3 follows the proof of Theorem 1, with the fact that $\Psi(B(\tilde{p}, A, \tilde{M})) \subset(B(p, A, M)$.

\section{ACKNOWLEDGEMENT}

I would like to thank the referee for his/her insightful and helpful comments.

\section{REFERENCES}

[A] M. Anderson, On the topology of complete manifolds of non-negative Ricci curvature, Topology, 29 Vol 1 (1990) 41-45. MR 91b:53041

[Be] M. Berger, Une borne inferieure pour le volume d'une variete Riemannienne en fonction du rayon d'injectivite, Ann. Inst. Fourier, Grenoble, 30 (1980), 259-265. MR 82b:53047

[BC] R. L. Bishop and R. J. Crittenden, Geometry of Manifolds, Academic Press, 1964. MR 29:6401

[CE] J. Cheeger and D. G. Ebin, Comparison Theorems in Riemannian Geometry, North Holland Mathematical Library, 9, 1975. MR 56:16538

[Cr] C. Croke, Some isoperimetric inequalities and eigenvalue estimates, Ann. Sci. Ecole Norm. Sup., 13 (1980) 419-435. MR 83d:58068

[C2] C. Croke, An isoembolic pinching theorem, Inventiones Mathematicae, 92 (1988) 385-387. MR 89e:53061

[D1] O. C. Durumeric, Manifolds with almost equal diameter and injectivity radius, Jour. of Differential Geometry, 19 (1984) 453-474. MR 86j:53061

[D2] O. C. Durumeric, Finiteness theorems, average volume and curvature, Amer. Jour. of Math., 111 (1989) 973-990. MR 91a:53063

[GKM] D. Gromoll, W. Klingenberg and W. Meyer, Riemannsche Geometrie im Grossen, Lecture notes in Mathematics No. 55, Springer-Verlag, 2nd Edition, 1975. MR 51:1651

[GW] D. Gromoll and J. Wolf, Some relations between the metric structure and the algebraic structure of the fundamental group in manifolds of nonpositive curvature, Bull. Amer. Math. Soc. 77(4) (1971) 545-552. MR 43:6841

[Gr] M. Gromov, Structures Metriques pour les Varietes Riemanniennes (J. LaFontaine and P. Pansu, eds.), CEDIC, Paris, 1981. MR 85e:53051 
[LY] H. B. Lawson and S. T. Yau, On compact manifolds of nonpositive curvature, Jour. of Diff. Geometry, 7 (1972), 211-228. MR 48:12402

[Mi] J. Milnor, A note on curvature and fundamental group, J. of Diff. Geom., 2 (1968), 1-7. MR 38:636

[P] A. Preismann, Quelques proprietes globales des espaces de Riemann, Comment Math. Helvetici, 15 (1943), 175-216.

[Y] T. Yamaguchi, Homotopy type finiteness theorems for certain precompact families of Riemannian manifolds, Proc. Amer. Math. Soc., 102, No. 3 (1988) 660-666. MR 89d:53088

Department of Mathematics, University of Iowa, Iowa City, Iowa 52242

E-mail address: odurumer@blue.weeg.uiowa.edu 